\title{
Midbrain/pons area ratio and clinical features predict the prognosis of Progressive Supranuclear Palsy
}

\section{CURRENT STATUS: ACCEPTED}

BMC Neurology $\triangle B M C$ series

shishuang cui

Shanghai Jiao Tong University Medical School Affiliated Ruijin Hospital

huawei ling

Shanghai Jiao Tong University Medical School Affiliated Ruijin Hospital

juanjuan du

Shanghai Jiao Tong University Medical School Affiliated Ruijin Hospital

yiqi lin

Shanghai Jiao Tong University Medical School Affiliated Ruijin Hospital

jing pan

Shanghai Jiao Tong University Medical School Affiliated Ruijin Hospital

haiyan zhou

Shanghai Jiao Tong University Medical School Affiliated Ruijin Hospital

gang wang

Shanghai Jiao Tong University Medical School Affiliated Ruijin Hospital

ying wang

Shanghai Jiao Tong University Medical School Affiliated Ruijin Hospital

qin xiao

Shanghai Jiao Tong University Medical School Affiliated Ruijin Hospital

jun liu

Shanghai Jiao Tong University Medical School Affiliated Ruijin Hospital

yuyan tan

Shanghai Jiao Tong University Medical School Affiliated Ruijin Hospital

shengdi chen

Shanghai Jiao Tong University Medical School Affiliated Ruijin Hospital 


\section{DOI:}

10.21203/rs.2.19508/v1

\section{SUBJECT AREAS}

Neurology Neurosurgery

\section{KEYWORDS}

progressive supranuclear palsy, survival analysis, M/P area ratio 
Abstract

Introduction: Progressive Supranuclear Palsy (PSP) is a rare movement disorder with poor prognosis. The natural history of PSP has been described in a few studies. This retrospective study aims to characterize the natural history of PSP and to find predictors of shorter survival and faster decline of activity of daily living.

Method: All patients recruited fulfilled the movement disorder society (MDS) clinical diagnostic criteria for PSP (MDS-PSP criteria) for probable and possible PSP with median 12 years. Data were obtained including age, gender, date of onset, age at onset (AAO), symptoms reported at first visit and followup, date of death and date of institutionalization. Magnetic resonance imaging was collected at the first visit. Endpoints were death and institutionalization. K-M method and Cox proportional hazard model were used to explore factors associated with early death and institutionalization.

Results: 59 patients fulfilled MDS-PSP criteria were enrolled in our study. 19 patients (32.2\%) had died and 31 patients $(52.5 \%)$ had institutionalized by the end of the follow-up. Predictors associated with poorer survival were late-onset PSP and decreased M/P area ratio. Predictors associated with earlier institutionalization were older AAO and decreased M/P area ratio.

Conclusion: Older $\mathrm{AAO}$ and decreased M/P area ratio were predictors for earlier dearth and institutionalization in PSP. The neuroimaging biomarker M/P area ratio was a predictor for prognosis in PSP.

Full-text

Due to technical limitations, full-text HTML conversion of this manuscript could not be completed. However, the manuscript can be downloaded and accessed as a PDF.

Figures 

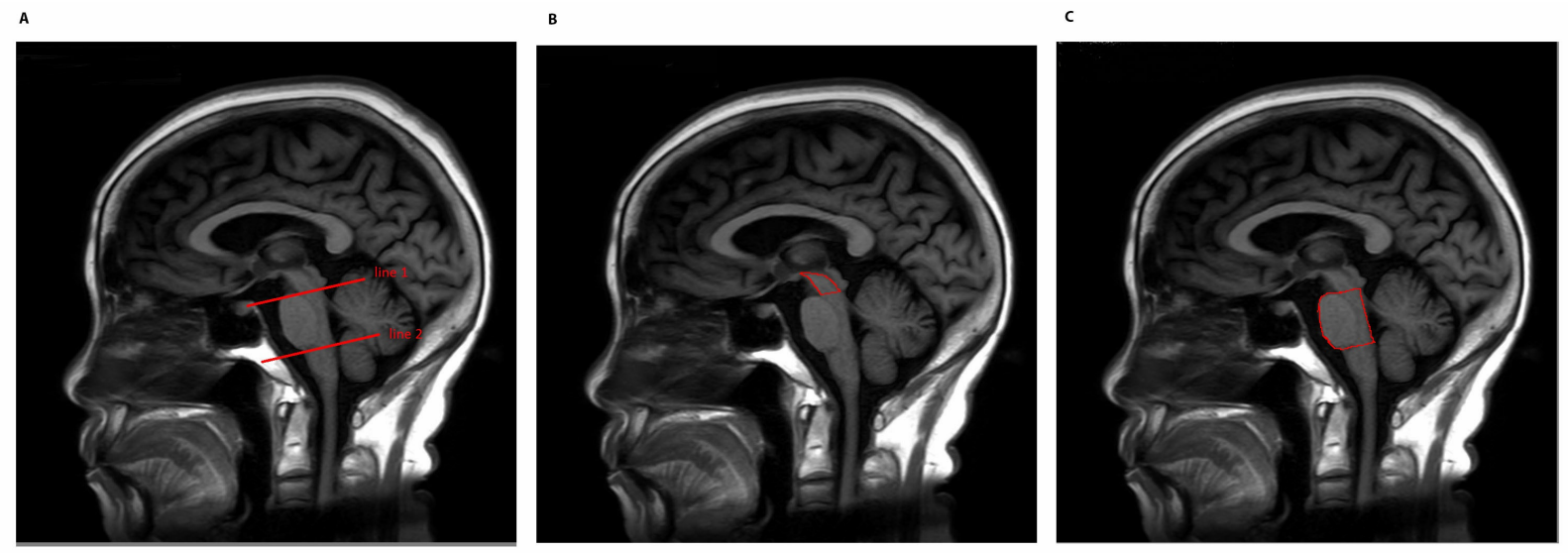

Figure 1

Sagittal T1-weighted MR images show midbrain area and pons area. (A) line 1 and line 2; (B) midbrain area; (C) pons area 